\title{
ANISOTROPIC NONLINEAR DIFFUSION WITH ABSORPTION: EXISTENCE AND EXTINCTION
}

\author{
ALAN V. LAIR and MARK E. OXLEY \\ Department of Mathematics and Statistics \\ Air Force Institute of Technology/EN(: \\ 2950 P Street \\ Wright-Patterson Air Force Base, Ohio 45433-7765
}

ISSA

(Received April 14, 1994 and in revised form October 19, 1994)

ABSTRACT. The authors prove that the nonlinear parabolic partial differential equation

$$
\frac{\partial u}{\partial t}=\sum_{\imath, j=1}^{n} \frac{\partial^{2}}{\partial x_{\imath} \partial x_{\jmath}} \varphi_{\imath \jmath}(u)-f(u)
$$

with homogeneous Dirichlet boundary conditions and a nonnegative initial condition has a nonnegative generalized solution $u$. They also give necessary and sufficient conditions on the constitutive functions $\varphi_{\imath \jmath}$ and $f$ which ensure the existence of a time $t_{0}>0$ for which $u$ vanishes for all $t \geq t_{0}$.

KEY WORDS AND PHRASES. Anisotropic nonlinear diffusion, finite extinction time, generalized solution.

1992 AMS SUBJECT CLASSIFIC:ATION CODES. 35K20, 35K55, 35K65.

\section{INTRODUCTION.}

We consider the nonlinear initial-boundary value problem

$$
\begin{aligned}
\frac{\partial u}{\partial t} & =\sum_{\imath, \jmath=1}^{n} \frac{\partial^{2}}{\partial x_{\imath} \partial x_{\jmath}} \varphi_{\imath \jmath}(u)-f(u) & & \text { in } Q_{\infty} \equiv \Omega \times(0, \infty) \\
\Phi(u) & =0 & & \text { on } \partial \Omega \times[0, \infty) \\
u(x, 0) & =u_{0}(x) \geq 0 & & \text { on } \bar{\Omega}
\end{aligned}
$$

in which $\Phi=\left(\varphi_{\imath \jmath}\right)$ is an $n \times n$ symmetric matrix and the domain $\Omega \subseteq \mathbf{R}^{n}$ is bounded. We assume that the functions $\varphi_{\imath j}$ and the nonnegative, nondecreasing function $f$ are in $C([0, \infty)) \cap C^{1}((0, \infty))$ and satisfy $f(0)=\varphi_{i y}(0)=0$. Furthermore, we assume that the matrix $\Phi^{\prime}(s)=\frac{d}{d s} \Phi(s)$ is positive definite on $(0, \infty)$ and there exists a positive function $\lambda \in C((0, \infty))$ such that

$$
\lambda(s)|\xi|^{2} \leq \sum_{i, j=1}^{n} \varphi_{\imath \jmath}^{\prime}(s) \xi_{2} \xi_{3} \leq \Lambda^{\prime}(s)|\xi|^{2}
$$

for $s>0, \xi \in \mathbf{R}^{n}$ where $\Lambda(s) \equiv \operatorname{tr}(\Phi(s))$, the trace of the matrix $\Phi(s)$, so that $\Lambda^{\prime}(s)=\operatorname{tr}\left(\Phi^{\prime}(s)\right)$. The main purpose of this article is to prove that the initial- boundary value problem (1.1) has a solution and to give necessary and sufficient conditions on the constitutive functions $\varphi_{\imath \jmath}$ and $f$ to 
ensure the existence of a finite extinction time (i.e., a time $t_{0}>0$ such that any solution $u$ satisfies $u(x, t)=0$ for all $\left.(x, t) \in \bar{\Omega} \times\left[t_{0}, \infty\right)\right)$.

Such problems have been considered for over two decades for the isotropic problem in which the matrix $\Phi$ is a scalar multiple of the identity matrix (i.e., $\Phi=\varphi \mathrm{I}$ ) . (Sec [1],[2],[3],[4],[5],[6],[7] and their references.) The main thust of these studies has been the determination of conditions on the functions $\varphi$ and $f$ which ensure the existence or nonexistence of a finite extinction time. For a single equation (see [2] for systems), the principal results can be summarized by those contained in [6] which contains all of the other results when homogeneous Dirichlet boundary conditions are specified. There it is shown that a sufficient condition to ensure the existence of a finite extinction time is that $(\varepsilon>0)$

$$
\int_{0}^{\varepsilon} \frac{d s}{\varphi(s)}<\infty \text { or } \int_{0}^{\varepsilon} \frac{d s}{f(s)}<\infty
$$

holds. Conversely, the authors prove that if it is known that a solution has a finite extinction time, then

$$
\int_{0}^{\varepsilon} \frac{d s}{\varphi(s)+f(s)}<\infty
$$

must hold.

For anisotropic diffusion, such results are virtually nonexistent. Indeed the authors know of no results in which either existence of a solution or existence of a finite extinction time for problem (1.1) has been treated. Other problems for special cases of (1.1) have been studied. For example, Kersner [8] demonstrates some properties of the solution to the Cauchy problem for the equation $u_{t}=\left(u^{m}\right)_{x x}+\left(u^{p}\right)_{y y}$.

It is well-known that classical solutions to (1.1) do not, in general, exist and hence weak solutions must be considered. We prove the existence of such a solution. Our definition of a weak, or generalized, solution is quite similar to that of Benilan et al [9]. In addition to existence of a solution, we give necessary and sufficient conditions for the existence of a finite extinction time. In particular, we show that if

$$
\int_{0}^{\varepsilon} \frac{d s}{f(s)}<\infty
$$

or if there exists some $m \in\{1,2, \ldots, n\}$ such that

$$
\int_{0}^{\varepsilon} \frac{d s}{\varphi_{m m}(s)}<\infty
$$

then any solution to our problem has a finite extinction time. On the other hand, we show that a necessary condition for the existence of a finite extinction time is

$$
\int_{0}^{\varepsilon} \frac{d s}{f(s)+\operatorname{tr}(\Phi(s))}<\infty
$$

These results contain all of those for the isotropic problem for which a bounded domain and Dirichlet boundary conditions are specified.

\section{STATEMENT OF MAIN RESULTS.}

Since problem (1.1) does not, in general, have a classical solution even in the isotropic case (see [4]), it is necessary to work with a weaker formulation of a solution. In the following, we give the definition of a generalized solution. It should be noted that our definition is similar to that of Benilan et al (see p. 218 of [9]). 
DEFINITION. The sequence of problems $\left(\Phi_{h}\right.$ is the matrix $\left.\left(\varphi_{\imath \jmath}^{h}\right)\right)$

$$
\begin{aligned}
\frac{\partial v_{k}}{\partial t} & =\sum_{l, j=1}^{n} \frac{\partial^{2}}{\partial x_{\imath} \partial x_{\jmath}} \varphi_{l_{j}}^{k}\left(v_{k}\right)-f_{k}\left(v_{k}\right) & & \text { in } Q_{\infty} \\
\Phi_{k}\left(v_{k}\right) & =0 & & \text { on } \partial \Omega \times[0, \infty) \\
v_{k}(x, 0) & =u_{0, k}(x) \geq 0 & & \text { on } \bar{\Omega}
\end{aligned}
$$

is called an approximating sequence of problems for the problem if the sequences of $C^{2}([0, \infty))$ functions $\left\{\varphi_{i \jmath}^{k}\right\}_{k=1}^{\infty}$ and $C^{1}([0, \infty))$ functions $\left\{f_{k}\right\}_{k=1}^{\infty}$ converge to $\varphi_{\imath \jmath}$, and $f$, respectively, uniformly on compact subsets of $[0, \infty)$ and the sequence $\left\{u_{0, k}\right\}$ converges to $u_{0}$ in $L_{\infty}(\Omega)$.

DEFINITION. A function $u \in L_{\infty}\left(Q_{\infty}\right)$ is a generalized solution of the initial-boundary value problem (1.1) if for each $T>0$, the lunction $u$ can be written as the weak $L_{2}\left(Q_{T}\right)$ limit of a sequence of classical solutions to an approximating sequence of problems for the problem (1.1).

The main purpose of this article is to prove the following three theorems.

THEOREM 1. (Exstence) Suppose $u_{0}$ is a nonnegative function continuous on $\bar{\Omega}$. Then the initial-boundary value problem (1.1) has a nonnegative generalized solution $u$ and $\|u(\cdot, t)\|_{\infty, \Omega} \leq$ $\left\|u_{0}\right\|_{\infty, \Omega}$ for all $t \geq 0$.

We are also interested in establishing both necessary and sufficient conditions on the matrix $\Phi$ and the function $f$ which ensure the existence of a finite extinction time for these weak solutions. The following two theorems are natural extensions of similar results for the isotropic case [6].

THEOREM 2. (Necessity) Let $u_{0}$ be a nontrivial nonnegative function continuous on $\bar{\Omega}$. If any nonnegative, bounded, generalized solution to the problem (1.1) has a finite extinction time, then (1.5) holds.

THEOREM 3. (Sufficiency) Let $u_{0}$ be a nonnegative function continuous on $\bar{\Omega}$. If either (1.3) holds or (1.4) holds for some $m \in\{1,2, \ldots, n\}$, then any nonnegative, bounded, generalized solution of (1.1) has a finite extinction time.

It should be noted that there is a gap between necessity and sufficiency which the authors have been unable to fill. That is, we show that a sufficienct condition to ensure the existence of a finite extinction time is to have either strong absorption (i.e., $f$ satisfies (1.3)) or fast diffusion in (at least) one direction (i.e., for some $m,(1.4)$ holds). However, necessity requires only that some combination of the absorption and diffusion be "fast" (i.e., (1.5) holds). Thus, suppose that one has absorption and diffusion terms for which the integrals in (1.3) and (1.4) are infinite for all $m=1, \ldots, n$ while inequality (1.5) holds. (Such functions do exist.) Does any solution to the resulting problem (1.1) have a finite extinction time? The authors have been unable to answer this question.

\section{PROOF OF EXISTENCE.}

PROOF OF THEOREM 1. Let $\left\{u_{0, k}\right\}$ be a sequence of $C_{0}^{\infty}(\Omega)$ functions converging uniformly on $\bar{\Omega}$ to $u_{0}$. Furthermore, we choose $u_{0, k}$ so that $\left\|u_{0, k}\right\|_{\infty, \Omega} \leq\left\|u_{0}\right\|_{\infty, \Omega} \equiv M$. Let $\left\{\varphi_{\imath \jmath}^{k}\right\}_{k=1}^{\infty}, 1 \leq$ $i, j \leq n$, and $\left\{f_{k}\right\}$ be sequences of functions such that $\varphi_{\imath \jmath}^{k} \in C^{2}([0, \infty))$, the matrix $\Phi_{k}^{\prime}(s)$ (i.e., the matrix $\left.\left(\frac{d}{d s} \varphi_{\imath \jmath}^{k}(s)\right)\right)$ is positive definite on compact subsets of $[0, \infty), f_{k} \in C^{1}([0, \infty))$ has a positive derivative everywhere and $\varphi_{\imath \jmath}^{k} \rightarrow \varphi_{i j}$ and $f_{k} \rightarrow f$ uniformly on compact subsets of $[0, \infty)$ as $k \rightarrow \infty$.

The existence of the sequences $\left\{\Phi_{k}\right\}$ and $\left\{f_{k}\right\}$ are fairly easy to demonstrate. To produce the sequence $\left\{\Phi_{k}\right\}$, we set $\Psi_{k}(s)=\Phi^{\prime}(1 / k)$ for $s \leq 1 / k$ and let $\Psi_{k}(s)=\Phi^{\prime}(s)$ for $s \geq 1 / k$. Let $\Phi_{k}(s)=$ $\int_{0}^{s} \Psi_{k}(\sigma) d \sigma$. Clearly, the sequence $\left\{\Phi_{k}\right\}$ has the required properties (except for smoothness). In 
particular. we note that

$$
\lambda\left(\frac{l}{h}\right)|\xi|^{2} \leq \xi^{l} \Phi_{h}^{\prime}(s) \xi \leq I^{\prime}\left(\frac{1}{h}\right)|\xi|^{2}
$$

for all $s \in[0, M]$ and $\xi \in \mathbf{R}^{n}$. An appropriate smooth approximation to this $\Phi_{k}$ will provide the required sequence. The sequence $\{/ h\}$ can be constructed similarly.

From Ladyzenskaja et al [10] (ree p. 157) we know that the sequence of initial-boundary value problems given by $(2.1)$ with $\Phi_{k}$ and $f_{h}$ as just defined has a unique nonnegative classical solution on $Q_{T}$ if $0<T<\infty$.

We now establish that a classical solution to (2.1) exists on $Q_{\infty}$. This is easy to do as follows: Let $w_{1}$ be a classical solution on $Q_{1}$ and define $u_{1}=w_{1}$ on $Q_{1}$ and $u_{1}=0$ elsewhere. Similarly, let $w_{2}$ be a classical solution on $Q_{2}$ and define $u_{2}=w_{2}$ on $Q_{2}$ and $u_{2}=0$ otherwise. Continue this process to produce a sequence $\left\{u_{m}\right\}$ for which $u_{m}=w_{m}$ on $Q_{m}$ and $u_{m}=0$ otherwise. Since the classical solution on $Q_{m}$ is unique, we get $u_{m}=u_{m+1}$ on $Q_{m}$. Furthermore, we get $0 \leq u_{m} \leq u_{m+1} \leq\left\|u_{0, k}\right\|_{\infty, \Omega} \leq M$ on $Q_{\infty}$ for all $m$.

Thus the sequence $\left\{u_{m}\right\}$ converges pointwise on $Q_{\infty}$. Let $v=\lim _{m \rightarrow \infty} u_{m}$ and notice that $v=u_{m}$ on $Q_{m}$ for all $m$. Thus, given any point in $Q_{\infty}, m$ may be chosen sufficiently large that $v=u_{m}$ at that point and thus $v$ is a (not necessarily unique) nonnegative classical solution to (2.1) on $Q_{\infty}$.

Since this classical solution obviously depends on $k$, we now let it be denoted by $v_{k}$ and observe that $\left\|v_{k}(\cdot, t)\right\|_{\infty, \Omega} \leq\left\|u_{0}\right\|_{\infty, \Omega}$ for all $k$ and $t \in[0, \infty)$. To obtain a generalized solution to our problem, we let $T \in(0, \infty)$ and note that we need only prove that the sequence $\left\{v_{k}\right\}$ has a subsequence $\left\{v_{k_{m}}\right\}$ which converges weakly in $L_{2}\left(Q_{T}\right)$. Then, after reindexing the sequences $\left\{\varphi_{i j}^{k}\right\}$, $\left\{f_{k}\right\}$ and $\left\{u_{0, k}\right\}$ replacing $k$ with $k_{m}$, we obtain the desired result. Thus, we let $T \in(0, \infty)$ and prove that the sequence $\left\{v_{k}\right\}$ has a subsequence $\left\{v_{k_{m}}\right\}$ which is weakly convergent in $L_{2}\left(Q_{T}\right)$. For ease of notation, we let $w=v_{k}$, multiply the differential equation in (2.1) by $w$ and integrate over $Q_{t}$. Integration by parts produces

$$
\frac{1}{2} \int_{\Omega} w^{2}(x, t) d x+\int_{Q_{t}}\left[\varphi_{t_{\jmath}}^{k}\right]^{\prime}(w) w_{x_{\mathrm{t}}} w_{x} d x d \tau+\int_{Q_{t}} w f_{k}(w) d x d \tau=\frac{1}{2} \int_{\Omega} u_{0, k}^{2} d x
$$

valid for all $t \in[0, T]$. Using $(3.1)$ and the positiveness of $f_{k}$, we get

$$
\int_{\Omega} w^{2}(x, t) d x \leq \int_{\Omega} u_{0, k}^{2} d x
$$

for all $t \in[0, T]$. Integrating over $[0, T]$, we have

$$
\int_{Q_{T}} w^{2} d x d t \leq T \int_{\Omega} u_{0, k}^{2} d x \leq M^{2} T|\Omega|
$$

which, in turn, produces

$$
\int_{Q_{T}} v_{k}^{2} d x d t \leq M^{2} T|\Omega|
$$

Since the right side of this inequality is bounded independently of $k$, the reflexivity of $L_{2}\left(Q_{T}\right)$ yields the existence of a subsequence of $\left\{v_{k}\right\}$ which converges weakly in $L_{2}\left(Q_{T}\right)$. This completes the proof of Theorem 1.

\section{PROOFS OF EXTINCTION RESULTS.}

PROOF OF THEOREM 2. Let $u$ be a nonnegative, bounded, generalized solution to (1.1). Let $t_{0}$ be the extinction time for $u$ with $0<t_{0}<\infty$ and let $x_{0} \in \Omega$ such that $u_{0}\left(x_{0}\right)>0$. Choose 
$r>0$ and $\delta>0$ so that $B \equiv\left\{x \in \Omega|| x-x_{0} \mid \leq r\right\} \subseteq \Omega$ and $u_{0}(x) \geq \delta$ for all $x \in B$. Let $h$ be the function defined by

$$
h(x)=\left\{\begin{array}{cl}
\exp \left(\frac{-\left|x-x_{0}\right|^{2}}{r^{2}-\left|x-x_{0}\right|^{2}}\right) & \text { if }\left|x-x_{0}\right|<r \\
0 & \text { if }\left|x-x_{0}\right| \geq r .
\end{array}\right.
$$

We note that $h$ satisfics $0 \leq h(x) \leq 1$ on $B, \int_{\Omega} h(x) d x<\infty$, and $|\nabla h|^{2} \leq K h$ on $\Omega$ for some constant $K$ depending only on the value of $r$. We define the sequence of nonnegative functions $\left\{a_{k}\right\}$ by $a_{k}(s) \equiv \Lambda_{k}(s)+f_{k}(s)$ where $\Lambda_{h}=\operatorname{tr}\left(\Phi_{k}\right)$ and the sequences $\left\{\Phi_{k}\right\},\left\{f_{k}\right\}$, and $\left\{u_{0, k}\right\}$ are defined as in the proof of Theorem 1 . Now let $\left\{u_{k}\right\}$ be a corresponding sequence of classical solutions to (2.1) which converges weakly in $L_{2}\left(Q_{t_{0}+1}\right)$ to $u$. We multiply (2.1) (with $v_{k}$ replaced by $u_{k}$ ) by $h$, divide by $a_{k}\left(u_{k}\right)+\varepsilon(\varepsilon>0)$ and integrate over $\Omega$. After integrating by parts, we get

$$
\begin{aligned}
\frac{d}{d t} \int_{\Omega} h \int_{0}^{u_{k}} \frac{d s}{a_{k}(s)+\varepsilon} d x= & -\int_{\Omega} \sum_{i, j=1}^{n}\left(\varphi_{\imath \jmath}^{k}\left(u_{k}\right)\right)_{x_{i}}\left(\frac{h}{a_{k}\left(u_{k}\right)+\varepsilon}\right)_{x_{j}} d x \\
& -\int_{\Omega} \frac{f_{k}\left(u_{k}\right) h}{a_{k}\left(u_{k}\right)+\varepsilon} d x \\
= & \int_{\Omega} \frac{a_{k}^{\prime}\left(u_{k}\right) h}{\left(a_{k}\left(u_{k}\right)+\varepsilon\right)^{2}} \nabla u_{k}^{T} \Phi_{k}^{\prime}\left(u_{k}\right) \nabla u_{k} d x \\
& -\int_{\Omega} \frac{1}{a_{k}\left(u_{k}\right)+\varepsilon} \nabla u_{k}^{T} \Phi_{k}^{\prime}\left(u_{k}\right) \nabla h d x \\
& -\int_{\Omega} \frac{f_{k}\left(u_{k}\right) h}{a_{k}\left(u_{k}\right)+\varepsilon} d x .
\end{aligned}
$$

We let $H$ be defined by

$$
H(x)=\left\{\begin{array}{cl}
\frac{|\nabla h(x)|}{h(x)^{1 / 2}} & \text { for }\left|x-x_{0}\right|<r \\
0 & \text { for }\left|x-x_{0}\right| \geq r .
\end{array}\right.
$$

We note that $H$ is continuous and bounded on $\bar{\Omega}$. (In fact, $0 \leq H(x) \leq K^{1 / 2}$ where the constant $K$ comes from the properties of $h$ above.) Using the fact that $h^{1 / 2} H=|\nabla h|$ on $\Omega$ and elementary estimates, the second integral on the right side of (4.1) may be estimated as

$$
\begin{aligned}
-\int_{\Omega} \frac{\nabla u_{k}^{T} \Phi_{k}^{\prime}\left(u_{k}\right) \nabla h}{a_{k}\left(u_{k}\right)+\varepsilon} d x \geq & -\int_{\Omega} \frac{1}{a_{k}\left(u_{k}\right)+\varepsilon}\left|\Phi_{k}^{\prime}\left(u_{k}\right) \nabla u_{k}\right||\nabla h| d x \\
= & -\int_{\Omega} \frac{h^{1 / 2}}{a_{k}\left(u_{k}\right)+\varepsilon}\left|\Phi_{k}^{\prime}\left(u_{k}\right) \nabla u_{k}\right| H d x \\
= & \int_{\Omega}\left(\frac{h^{1 / 2}\left|\Phi_{k}^{\prime}\left(u_{k}\right) \nabla u_{k}\right|}{a_{k}\left(u_{k}\right)+\varepsilon}-\frac{H}{2}\right)^{2} d x \\
& -\int_{\Omega} \frac{\left|\Phi_{k}^{\prime}\left(u_{k}\right) \nabla u_{k}\right|^{2} h}{\left(a_{k}\left(u_{k}\right)+\varepsilon\right)^{2}} d x-\frac{1}{4} \int_{\Omega} H^{2} d x \\
\geq & -\int_{\Omega} \frac{\left|\Phi_{k}^{\prime}\left(u_{k}\right) \nabla u_{k}\right|^{2} h}{\left(a_{k}\left(u_{k}\right)+\varepsilon\right)^{2}} d x-\frac{1}{4} \int_{\Omega} H^{2} d x
\end{aligned}
$$

We now substitute this expression into (4.1) and use the fact that $f_{k}\left(u_{k}\right) \leq a_{k}\left(u_{k}\right)+\varepsilon$ to obtain

$$
\begin{aligned}
\frac{d}{d t} \int_{\Omega} h \int_{0}^{u_{k}} \frac{d s}{a_{k}(s)+\varepsilon} d x \geq & \int_{\Omega} \frac{a_{k}^{\prime}\left(u_{k}\right) h}{\left(a_{k}\left(u_{k}\right)+\varepsilon\right)^{2}} \nabla u_{k}^{T} \Phi_{k}^{\prime}\left(u_{k}\right) \nabla u_{k} d x \\
& -\int_{\Omega} \frac{\left|\Phi_{k}^{\prime}\left(u_{k}\right) \nabla u_{k}\right|^{2} h}{\left(a_{k}\left(u_{k}\right)+\varepsilon\right)^{2}} d x-\frac{1}{4} \int_{\Omega} H^{2} d x-\int_{\Omega} h d x .
\end{aligned}
$$


We use the positive definiteness of $\Phi_{k}^{\prime}$, inequality (3.1), and the inequality $a_{k}^{\prime}(s) \geq \Lambda_{k}^{\prime}(s)$ in $(4.2)$ to get (The argument $u_{k}$ has been suppressed for ease of reading.)

$$
\begin{aligned}
\frac{d}{d t} \int_{\Omega} \int_{0}^{u_{h}} \frac{h d s}{a_{k}(s)+\varepsilon} d x & \geq \int_{\Omega} \frac{h}{\left(a_{h}+\varepsilon\right)^{2}} \nabla u_{k}^{T}\left(\Phi_{k}^{\prime}\right)^{1 / 2}\left[a_{k}^{\prime} I-\Phi_{k}^{\prime}\right]\left(\Phi_{k}^{\prime}\right)^{1 / 2} \nabla u_{k} d x-\int_{\Omega} H^{2} d x-\int_{\Omega} h d x \\
& \geq-\frac{1}{4} \int_{\Omega} I^{2} d x-\int_{\Omega} h d x \equiv-K_{0}
\end{aligned}
$$

Integrating this inequality over the interval $[0, t]$ produces

$$
\int_{\Omega} \int_{0}^{u_{k}(x, t)} \frac{h(x) d s}{\Lambda_{k}(s)+f_{k}(s)+\varepsilon} d x \geq \int_{\Omega} \int_{0}^{u_{0, k}(\alpha)} \frac{h(x) d s}{\Lambda_{k}(s)+f_{k}(s)+\varepsilon} d x-\kappa_{0} t
$$

for all $0 \leq t<\infty$. Thus, integrating this over the interval $\left[t_{0}, t_{0}+1\right]$, produces

$$
\int_{t_{0}}^{t_{0}+1} \int_{\Omega} \int_{0}^{u_{k}(x, t)} \frac{h(x) d s}{\Lambda_{k}(s)+f_{k}(s)+\varepsilon} d x d t \geq \int_{\Omega} \int_{0}^{u_{0, k}(x)} \frac{h(x) d s}{\Lambda_{k}(s)+f_{k}(s)+\varepsilon} d x-K_{0}^{\prime}\left(t_{0}+1\right) .
$$

Since for fixed $k$ and fixed $\varepsilon>0$, the function $F$ defined by

$$
F(\sigma)=\int_{0}^{\sigma} \frac{d s}{\Lambda_{k}(s)+f_{k}(s)+\varepsilon}
$$

.is concave, Jensen's inequality (see [11] p. 202) may be applied to the left side of (4.3) to get

$$
|\Omega| \int_{0}^{U_{k}\left(t_{0}\right)} \frac{d s}{\Lambda_{k}(s)+f_{k}(s)+\varepsilon} \geq \int_{\Omega} h(x) \int_{0}^{u_{0, k}(x)} \frac{d s}{\Lambda_{k}(s)+f_{k}(s)+\varepsilon} d x-K_{0}\left(t_{0}+1\right) .
$$

where

$$
U_{k}\left(t_{0}\right)=\frac{1}{|\Omega|} \int_{t_{0}}^{t_{0}+1} \int_{\Omega} h(x) u_{k}(x, t) d x d t
$$

We now let $k$ approach $\infty$. The weak convergence of $\left\{u_{k}\right\}$ to $u$, the $L_{\infty}(\Omega)$ convergence of $\left\{u_{0, k}\right\}$ to $u_{0}$ as well as the uniform convergence of $\Lambda_{k}$ and $f_{k}$ to $\Lambda$ and $f$, respectively, produces

$$
|\Omega| \int_{0}^{U\left(t_{0}\right)} \frac{d s}{\Lambda(s)+f(s)+\varepsilon} \geq \int_{\Omega} h(x) \int_{0}^{u_{0}(x)} \frac{d s}{\Lambda(s)+f(s)+\varepsilon} d x-K_{0}\left(t_{0}+1\right) .
$$

where

$$
U\left(t_{0}\right)=\frac{1}{|\Omega|} \int_{t_{0}}^{t_{0}+1} \int_{\Omega} h(x) u(x, t) d x d t .
$$

Furthermore, since $t_{0}$ is the extinction time for $u$, we have $u(x, t)=0$ for almost all $(x, t) \in$ $\Omega \times\left[t_{0}, \infty\right)$. Therefore, the last inequality yields

$$
\int_{\Omega} h(x) \int_{0}^{u_{0}(x)} \frac{d s}{\Lambda(s)+f(s)+\varepsilon} d x \leq K_{0}\left(t_{0}+1\right) .
$$

By our choice of the set $B$ and the function $h$, this inequality produces

$$
\int_{B} h(x) \int_{0}^{\delta} \frac{d s}{\Lambda(s)+f(s)+\varepsilon} d x \leq \int_{B} h(x) \int_{0}^{u_{0}(x)} \frac{d s}{\Lambda(s)+f(s)+\varepsilon} d x \leq K_{0}\left(t_{0}+1\right)
$$

and hence

$$
\int_{0}^{\delta} \frac{d s}{\Lambda(s)+f(s)+\varepsilon} \leq \frac{K_{0}\left(t_{0}+1\right)}{\int_{B} h(x) d x}<\infty .
$$

Now let $\varepsilon \downarrow 0$ and the proof of Theorem 2 is complete.

PROOF OF THEOREM 3. Suppose $0 \leq u_{0} \leq M$. If (1.3) holds, then the proof of the existence of a finite extinction time is quite similar to that of the isotropic case (see Theorem 1 of [5]) and is therefore omitted. 
Thus, suppose (1.1) holds for some $m$. For ease of notation, we let $\psi=\varphi_{m m}$. Choose $r>0$ so that $\bar{\Omega} \subseteq\left\{x \in \mathbf{R}^{n}|| x_{2} \mid<r, 1 \leq i \leq n\right\}$ and let $z_{0}$ bc a $\left({ }^{\infty}(\mathbf{R})\right.$ function such that $z_{0}\left(x_{m}\right)=M$ for $\left|x_{m}\right|<r$ and $z_{0}(-r-1)=z_{0}(r+1)=0$. Let $z$ be a mild solution (as in [9] and [12]) to the initial-boundary value problem

$$
\begin{array}{lll}
\frac{\partial}{\partial t} z=\frac{\partial^{2}}{\partial x_{m}^{2}} \psi^{\prime}(z) & \text { for }\left|x_{m}\right|<r+1, & t>0 \\
z(-r-1 . t)=0 & \text { for } & t \geq 0 \\
z(r+1 . t)=0 & \text { for } & t \geq 0 \\
z\left(x_{m}, 0\right)=z_{0}\left(x_{m}\right) & \text { for }\left|x_{m}\right| \leq r+1 & .
\end{array}
$$

From [1], we know that $z$ has a finite extinction time, $T_{0}$. We shall show that $u=0$ a.e. for $t \geq T_{0}$. Thus let $T \in\left(T_{0}, \infty\right)$. Since $u$ is a generalized solution of $(1.1)$, there exist sequences $\left\{\varphi_{i j}^{k}\right\}_{k=1}^{\infty}$ and $\left\{f_{k}\right\}$ of well-behaved functions converging uniformly on compact subsets of $[0, \infty)$ to $\varphi_{i j}, 1 \leq i, j \leq n$, and to $f$, respectively, such that the sequence $\left\{v_{k}\right\}$ of classical solutions to (2.1) converges weakly in $L_{2}\left(Q_{T}\right)$ to the solution $u$. Let $z_{k}$ be the unique classical solution of $\left(\psi_{k} \equiv \varphi_{m m}^{k}\right)$

$$
\begin{array}{lll}
\frac{\partial}{\partial t} z_{k}=\frac{\partial^{2}}{\partial x_{m}^{2}} \psi_{k}\left(z_{k}\right) & \text { for }\left|x_{m}\right|<r+1, & t>0 \\
z_{k}(-r-1, t)=0 & \text { for } & t \geq 0 \\
z_{k}(r+1, t)=0 & \text { for } & t \geq 0 \\
z_{k}\left(x_{m}, 0\right)=z_{0}\left(x_{m}\right) & \text { for }\left|x_{m}\right| \leq r+1, &
\end{array}
$$

From Sacks [12] (see Proposition 2.1 of [12]), we know that $z_{k} \rightarrow z$ in $C\left([0, T] ; L^{1}(-r-1, r+1)\right.$ ). Also, since $z_{k}$ satisfies

$$
\begin{aligned}
L_{k} z_{k}=0 & \leq f_{k}\left(v_{k}\right)=L_{k} v_{k} & & \text { in } Q_{T} \\
v_{k} & \leq z_{k} & & \text { on } \partial \Omega \times[0, T] \\
v_{k}(x, 0) & \leq z_{0}(x) & & \text { on } \bar{\Omega}
\end{aligned}
$$

where $L_{k}$ is the operator defined by

$$
L_{k} w \equiv \sum_{i, j=1}^{n} \frac{\partial^{2}}{\partial x_{\imath} \partial x_{\jmath}} \varphi_{\imath \jmath}^{k}(w)-\frac{\partial w}{\partial t}
$$

the results from Protter and Weinberger [13] (see pp. 187-188) yield $v_{k} \leq z_{k}$ on $Q_{T}$ for all $k$. Hence

$$
\int_{T_{0}}^{T} \int_{\Omega} v_{k}(x, t) d x \leq \int_{T_{0}}^{T} \int_{\Omega} z_{k}\left(x_{m}, t\right) d x
$$

We now let $k \rightarrow \infty$ to get

$$
\int_{T_{0}}^{T} \int_{\Omega} u(x, t) d x \leq \int_{T_{0}}^{T} \int_{\Omega} z\left(x_{m}, t\right) d x .
$$

Thus, since $z$ vanishes for $t \geq T_{0}$, we must have $u=0$ a.e. on $\Omega \times\left[T_{0}, T\right]$. Since $T$ was chosen arbitrarily from $\left(T_{0}, \infty\right)$, we must have $u=0$ a.e. on $\Omega \times\left[T_{0}, \infty\right)$. This completes the proof of Theorem 3.

ACKNOWLEDGEMENT. The authors wish to thank the (unknown) referee for pointing out errors in the original manuscript. 


\section{REFERENCES}

1. DIAZ, G. and Diaz, I. Finite extinction time for a class of non-linear parabolic equations, Comm. Partial Differential liquation $\underline{4}$ (1979), 1213-12:31.

2. FRIEDMAN, A. and HERRERO, M.A. Extinction and positivity for a system of semilinear parabolic variational inequalities, J. Math. Anal. Appl. 167 (1992), 167-175.

3. KALASHNIKOV, A.S. The propagation of disturbances in problems of non-linear heat conduction with absorption, USSR Comp. Math. and Math. Phys. 14 (1974), 70-85.

4. KERSNER, R. Nonlinear heat conduction with absorption: space localization and extinction in finite time, SIAM J. Appl. Math. $\underline{43}$ (1983), 1274-1285.

5. LAIR, A.V. Finite extinction time for solutions of nonlinear parabolic equations, Nonlinear Anal. 21 (1993), 1-8.

6. LAIR, A.V. and OXLEY, M.E. Extinction in finite time of solutions to nonlinear absorptiondiffusion equations, J. Math. Anal. Appl. 182 (1994), 857-866.

7. LAIR, A.V. and OXLEY, M.E. Finite extinction time for a nonlinear parabolic Neumann boundary value problem, Applicable Anal. (to appear)

8. KERSNER, R. On anisotropic diffusion, Semigroups, Theory and Applications, Vol I, 164168, Pitman Res. Notes Math. Ser., 141, Longman Sci. Tech., Harlow, New York, 1986

9. BENILAN, P., CRANDALL, M.G. and SACKS, P. Some $L^{1}$ existence and dependence results for semilinear elliptic equations under nonlinear boundary conditions, Appl. Math. and Opt. 17 (1988), 203-224.

10. LADYZENSKAJA, O.A.,SOLONNIKOV, V.A. and URALCEVA, N.N. Linear and Quasilinear Equations of Parabolic Type, AMS Transl. Math. Mono. 23, Providence, 1968.

11. HEWITT, E. and STROMBERG, K. Real and Abstract Analysis, Springer-Verlag, New York, 1969.

12. SACKS, P.E. Universal decay in nonlinear parabolic equations, Nonlinear Anal. 12 (1988), 1123-1136.

13. PROTTER, M.H. and WEINBERGER, H.F. Maximum Principles in Differential Equations, Prentice-Hall, Englewood Cliffs, NJ, 1967. 


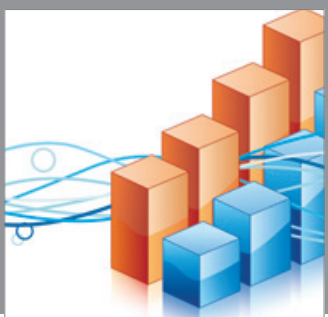

Advances in

Operations Research

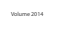

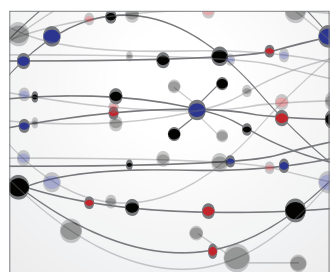

\section{The Scientific} World Journal
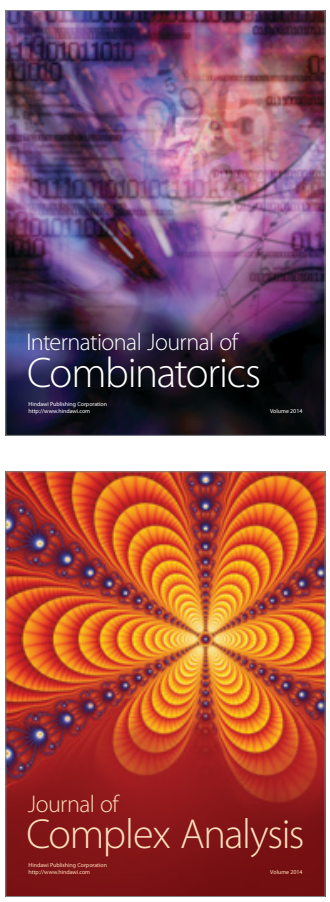

International Journal of

Mathematics and

Mathematical

Sciences
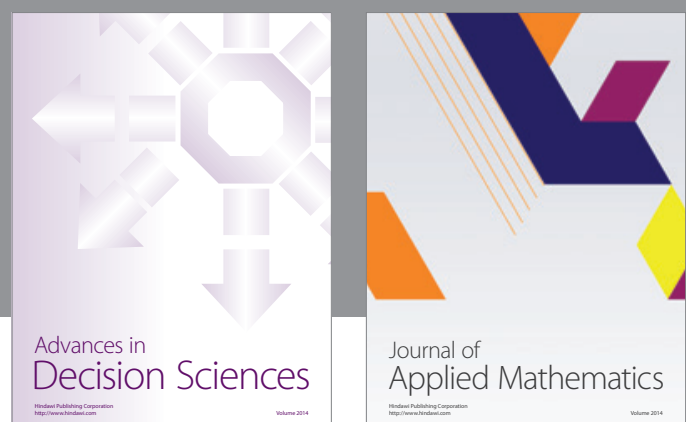

Journal of

Applied Mathematics
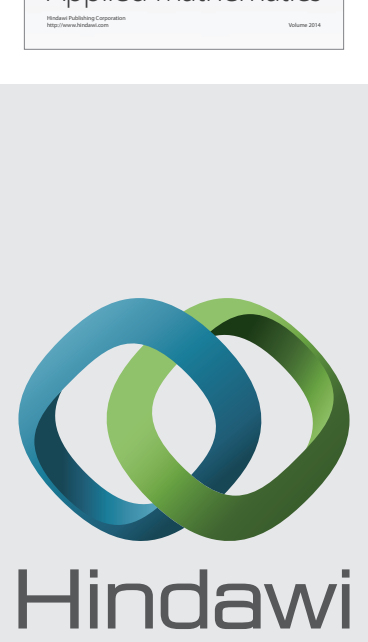

Submit your manuscripts at http://www.hindawi.com
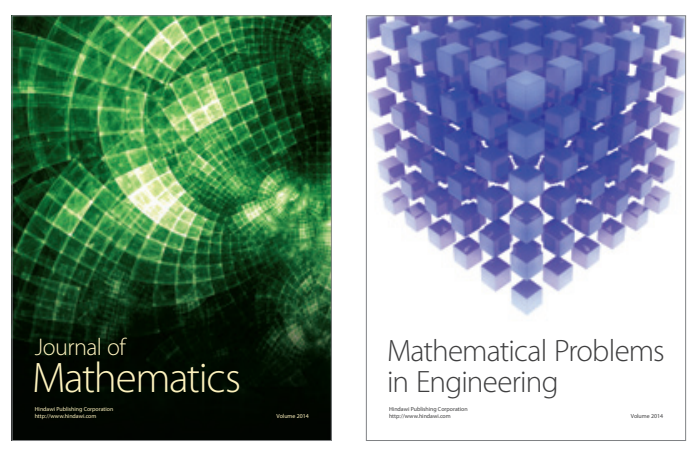

Mathematical Problems in Engineering
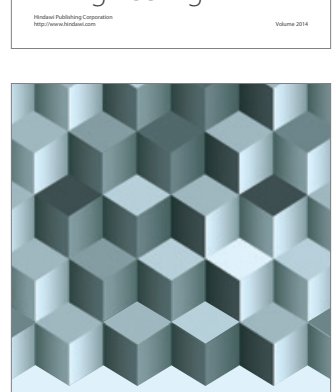

Journal of

Function Spaces
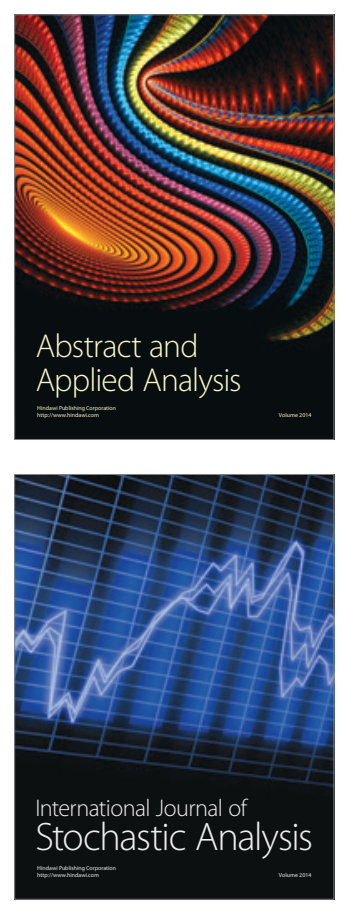

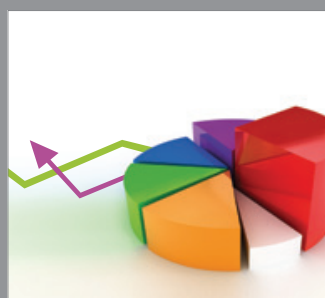

ournal of

Probability and Statistics

Promensencen
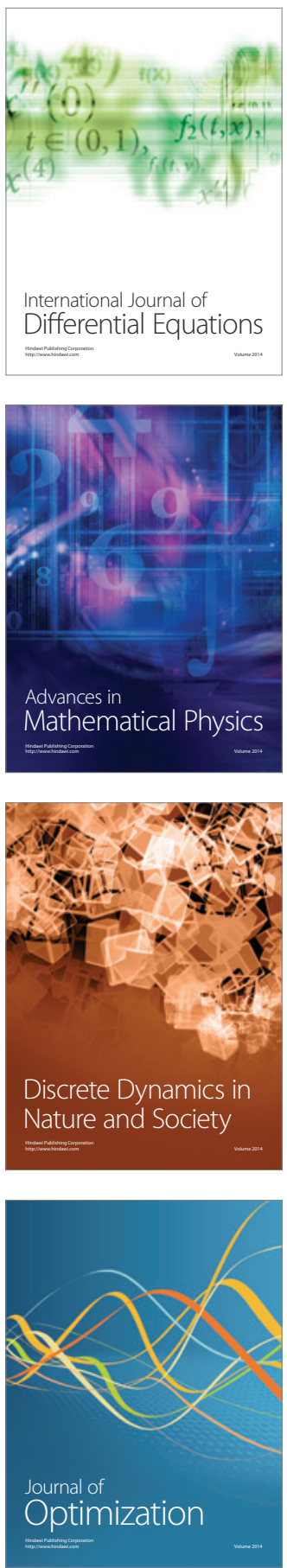